\title{
Editorial
}

\section{Special issue with papers from the ESLAB 2008 Symposium on 'Cosmic Cataclysms and Life'}

\author{
N. André'*, B.H. Foing' and C.S. Cockell ${ }^{2}$ \\ ${ }^{1}$ Research and Science Support Department, European Space Agency, Keplerlaan 1, 2200 AG Noordwijk, The \\ Netherlands \\ e-mail: Nicolas.Andre@cesr.fr; Bernard.Foing@esa.int \\ ${ }^{2}$ Center for Earth, Planetary, Space and Astronomical Research, Open University, Milton Keynes MK7 6AA, UK \\ e-mail:C.S.Cockell@open.ac.uk \\ *Current address Centre d'Etude Spatiale des Rayonnements, CNRS/Université Paul Sabatier, 9 avenue du colonel \\ Roche, 31028 Toulouse, France
}

The question of the origin of and conditions for life on Earth as a result of a succession of cosmic events following the Big Bang and the formation of stars, galaxies and planets, is fascinating and of wide interest. Answering this fundamental question requires an improved understanding of various biological, chemical, physical, geological, planetary and cosmic phenomena as well as the relationships among them. Since the advent of the space age, this multidisciplinary quest has benefitted from observations obtained by a number of missions in the fields of astrophysics, heliophysics, planetary sciences and Earth observation, with a growing number of directly or indirectly astrobiology-related missions to be launched in the 21 st century.

A number of cataclysms have occurred in the history of the Universe and the Solar System. The ESLAB Symposium on Cosmic Cataclysms and Life was held at the European Space Agency (ESA) European Space Research Institute (ESRIN) in Frascati, Italy, 10-14 November 2008. For more than 40 years, the Research and Scientific Support Department of ESA has organized the annual ESLAB symposium - always on a topic connected to the work of the Science and Robotic Exploration Directorate, and with the aim of enhancing interdisciplinary exchanges. This 42nd ESLAB on Cosmic Cataclysms and Life reflected the interest of the Directorate in the search for other habitable worlds, the critical effect cataclysms have had on these worlds and on the emergence and survival of life on Earth, and possibly elsewhere.

ESA plays a leading role in developing space missions and research opportunities for the understanding of many aspects of these cataclysms, and their potential effects on life to adapt to challenges on Earth and in space.

Among other missions, the Integral and XMM-Newton observatories look at gamma- and X-ray signatures of these cataclysms, which manifest during the evolution of stars throughout their lives, from birth to death. The infrared ISO and the Herschel observatories observe star- and planet-formation processes, whereas Hipparcos, Corot 
and the future Gaia observatory place our own planetary system into the overall context of planetary formation and provide important information on the likelihood of finding other habitable planetary systems. Closer to Earth, the SOHO, Ulysses and Cluster missions scrutinize the activity of the Sun and the violent effects of its perturbations on the heliosphere and the Earth's magnetosphere, while planetary missions such as Giotto, Mars Express, SMART-1, Venus Express, Rosetta and the future BepiColombo search for evidence of their effects on other bodies in our Solar System and contrast the environmental conditions when life first arose on Earth.

Sessions organized during the Symposium covered such topics as the Big Bang, violent processes in star formation and the synthesis of molecules, planetary formation and the role of collisions, formation of the Moon and its influence on Earth, the Late Heavy Bombardment and the role of impacts in inhibiting, transporting or threatening life, hazards from the Sun, Stars and the Universe, cosmic habitability and the fate of the Earth. Historical, outreach and educational aspects of cosmic cataclysms were also addressed during the Symposium. The best example of a cataclysm related to impact is the extinction of dinosaurs, but the most dramatic cosmic impact in recent history is the Tunguska event. For the 100th anniversary of the event, leading Tunguska expert Professor Longo, from the University of Bologna, was invited to give a public lecture on 'The Tunguska mystery, one century later', providing us with a good illustration on the need for laboratory and field research to complement space-based studies in astrobiology.

The Symposium programme included 43 talks, including 18 invited talks, which gave an overview of the broad science themes mentioned above. The full programme can be downloaded at: http://www.congrex.nl/08C16/. This special issue of International Journal of Astrobiology contains 10 refereed papers and abstracts of papers (oral contributions and posters) that were all presented at the Symposium.

The Organizers of the Symposium would like finally to acknowledge the Scientific Programme Committee (Jean-Charles Augereau, Willy Benz, Agustin Chicarro, Charles Cockell, Bernard Foing, Lisa Kaltenegger, Helmut Lammer, René Liseau, Hermann Opgenoorth, Günther Wuchterl, and Frances Westall) for their enthusiasm and recommendations, and the Local Organizing Committee (Clare Bingham, Malcolm Fridlund, Olivier Witasse, Dieter Isakeit and Carmen Comparetto) for making this Symposium such an interactive and successful week. 